\title{
Infrared fixed point structure in the minimal supersymmetric standard model with baryon and lepton number violation
}

\author{
B. Ananthanarayan \\ Centre for Theoretical Studies, Indian Institute of Science, Bangalore 560 012, India \\ P. N. Pandita \\ Department of Physics, North-Eastern Hill University, Shillong 793 022, India
}

\begin{abstract}
We study in detail the renormalization group evolution of Yukawa couplings and soft supersymmetry breaking trilinear couplings in the minimal supersymmetric standard model with baryon and lepton number violation. We obtain the exact solutions of these equations in a closed form, and then depict the infrared fixed point structure of the third generation Yukawa couplings and the highest generation baryon and lepton number violating couplings. Approximate analytical solutions for these Yukawa couplings and baryon and lepton number violating couplings, and the soft supersymmetry breaking couplings are obtained in terms of their initial values at the unification scale. We then numerically study the infrared fixed surfaces of the model, and illustrate the approach to the fixed points.
\end{abstract}

\section{INTRODUCTION}

Considerable attention has been focused on the infrared fixed point behavior [1] of the standard model (SM) and its extensions, especially the minimal supersymmetric standard model (MSSM). This is because there may be a stage of unification beyond the SM, and if so, it then becomes important to perform the radiative corrections in determining all the dimension $\leqslant 4$ terms in the Lagrangian. This can be achieved by using the renormalization group equations to find the values of parameters at the electroweak scale, given their values at the unification scale. As such, much effort has been devoted to the study of the evolution of various dimensionless Yukawa couplings in the SM, and its minimal supersymmetric extension, the MSSM. Using the renormalization group evolution, one can relate the Yukawa couplings to the gauge couplings via the Pendleton-Ross infrared stable fixed point (IRSFP) for the top-quark Yukawa coupling $[2,3]$, or via the quasi-fixed-point behavior [4]. The predictive power of the SM and its supersymmetric extensions may, thus, be enhanced if the renormalization group (RG) running of parameters is dominated by infrared stable fixed points (IRSFPs). These parameters (Yukawa couplings, ratios of Yukawa couplings to gauge couplings, etc.) do not attain fixed point values at the weak scale, the range between the grand unified theory (GUT) scale and the weak scale being too small for them to closely approach the fixed point. Nevertheless, the couplings may be determined by the quasifixed point behavior [4] where the value of the coupling at the weak scale is independent of its value at the GUT scale, provided the coupling at the GUT scale is large.

In supersymmetric theories there are superpartners of the ordinary particles in the spectrum, due to which there are additional Yukawa couplings [5] in these models which lead to baryon number $(B)$ or lepton number $(L)$ violation. Often, a discrete symmetry [6] called $R$-parity $\left(R_{p}\right)$ is introduced to eliminate these $B$ and $L$ violating Yukawa couplings. However, the assumption of $R_{p}$ conservation in MSSM appears to be ad hoc, since it is not required for the internal consistency of the model. Considerable attention has, thus, recently been focused on the study of the renormalization group evolution of Yukawa couplings, including the baryon and lepton number violating couplings, of the MSSM [7-10]. This includes the study of quasi-fixed-point behavior as well as the true infrared fixed points of the different Yukawa couplings, and the analysis of their stability. It has been shown that in the Yukawa sector of the minimal supersymmetric standard model with baryon and lepton number violation there is only one infrared stable fixed point. This corresponds to nontrivial fixed point for the top- and bottom-quark Yukawa couplings and the $B$ violating coupling $\lambda_{233}^{\prime \prime}$, and a trivial one for the $\tau$-Yukawa coupling and the $L$ violating coupling $\lambda_{233}$. It was shown that all other fixed points are either unphysical or unstable in the infrared region. Similarly, fixed points were obtained for the corresponding soft supersymmetry breaking trilinear couplings as well [9].

The purpose of the present paper is twofold. First, we study the renormalization group evolution in the minimal supersymmetric standard model with baryon and lepton number violation in order to obtain the exact as well as the approximate analytical solutions for the Yukawa couplings and the soft supersymmetry breaking couplings at the weak scale given their initial values at the ultraviolet (UV) or the GUT scale. Second, we study the renormalization group flow of such a system, and determine the infrared fixed surfaces and infrared fixed points toward which the renormalization group (RG) flow is attracted.

The plan of the paper is as follows. In Sec. II we describe the renormalization group equations for the minimal supersymmetric standard model with baryon and lepton mumber violation involving the highest generations. We obtain the exact solutions for the RG equations, and describe the infrared fixed points for the third generation Yukawa couplings and the highest generation baryon and lepton number violating couplings. Here we also study the corresponding RG 
equations for the soft supersymmetry breaking trilinear couplings, and describe their infrared fixed points. Section III is devoted to the study of the approximate analytical solutions of the RG equations for the Yukawa couplings and the soft supersymmetry breaking trilinear couplings. In Sec. IV we carry out a detailed numerical study of the infrared attractive fixed surfaces, and present their two dimensional projections to demonstrate the existence of a strongly attractive fixed point. In Sec. V we present the summary and conclusions.

\section{RENORMALIZATION GROUP EQUATIONS AND INFRARED FIXED POINTS}

\section{A. Infrared fixed points for Yukawa couplings}

In this section we recall some of the basic features of the renormalization group evolution in the minimal supersymmetric standard model with baryon and lepton number violation, and obtain the infrared fixed points of the Yukawa couplings and the soft supersymmetry breaking trilinear couplings of the model. The superpotential of the model is written as

$$
\begin{aligned}
W= & \left(h_{U}\right)_{a b} Q_{L}^{a} \bar{U}_{R}^{b} H_{2}+\left(h_{D}\right)_{a b} Q_{L}^{a} \bar{D}_{R}^{b} H_{1}+\left(h_{E}\right)_{a b} L_{L}^{a} \bar{E}_{R}^{b} H_{1} \\
& +\mu H_{1} H_{2},
\end{aligned}
$$

where $L, Q, \bar{E}, \bar{D}, \bar{U}$ denote the lepton and quark doublets, and anti-lepon singlet, d-type anti-quark singlet and u-type anti-quark singlet, respectively. In Eq. (1), $\left(h_{U}\right)_{a b},\left(h_{D}\right)_{a b}$ and $\left(h_{E}\right)_{a b}$ are the Yukawa coupling matrices, with $a, b, c$ as the generation indices. Gauge invariance, supersymmetry and renormalizability allow the addition of the following $L$ and $B$ violating terms to the MSSM superpotential (1):

$$
\begin{aligned}
W_{L} & =\frac{1}{2} \lambda_{a b c} L_{L}^{a} L_{L}^{b} \bar{E}_{R}^{c}+\lambda_{a b c}^{\prime} L_{L}^{a} Q_{L}^{b} \bar{D}_{R}^{c}+\mu_{i} L_{i} H_{2}, \\
W_{B} & =\frac{1}{2} \lambda_{a b c}^{\prime \prime} \bar{D}_{R}^{a} \bar{D}_{R}^{b} \bar{U}_{R}^{c},
\end{aligned}
$$

respectively. In this paper we shall consider, apart from the Yukawa couplings in Eq. (1), the dimensionless Yukawa couplings $\lambda_{a b c}, \quad \lambda_{a b c}^{\prime}$ and $\lambda_{a b c}^{\prime \prime}$ only, and ignore the dimensionful couplings $\mu$ and $\mu_{i}$. The couplings $\lambda_{a b c}$ and $\lambda_{a b c}^{\prime \prime}$ are antisymmetric in their first two indices due to $S U(2)_{L}$ and $S U(3)_{C}$ group structures, respectively. Corresponding to the terms in the superpotentials (1), (2) and (3), there are soft supersymmetry breaking trilinear terms which can be written as

$$
\begin{aligned}
-V_{\text {soft }}= & {\left[\left(A_{U}\right)_{a b}\left(h_{U}\right)_{a b} \widetilde{Q}_{L}^{a} \widetilde{\bar{U}}_{R}^{b} H_{2}+\left(A_{D}\right)_{a b}\left(h_{D}\right)_{a b} \widetilde{Q}_{L}^{a} \widetilde{\bar{D}}_{R}^{b} H_{1}\right.} \\
& \left.+\left(A_{E}\right)_{a b}\left(h_{E}\right)_{a b} \widetilde{L}_{L}^{a} \widetilde{\bar{E}}_{R}^{b} H_{1}\right] \\
& +\left[\frac{1}{2}\left(A_{\lambda}\right)_{a b c} \lambda_{a b c} \widetilde{L}_{L}^{a} \widetilde{L}_{L}^{b} \widetilde{\bar{E}}_{R}^{c}+\left(A_{\lambda^{\prime}}\right)_{a b c} \lambda_{a b c}^{\prime} \widetilde{L}_{L}^{a} \widetilde{Q}_{L}^{b} \widetilde{\bar{D}}_{R}^{c}\right] \\
& +\left[\frac{1}{2}\left(A_{\lambda^{\prime \prime}}\right)_{a b c} \lambda_{a b c}^{\prime \prime} \widetilde{\bar{D}}_{R}^{a} \widetilde{\bar{D}}_{R}^{b} \widetilde{\bar{U}}_{R}^{c}\right],
\end{aligned}
$$

where a tilde denotes the scalar component of the chiral superfield, and the notation for the scalar component of the Higgs superfield is the same as that of the corresponding superfield. In addition there are soft supersymmetry breaking gaugino mass terms with the masses $M_{i}$ with $i=1,2,3$, corresponding to the gauge groups $U(1)_{Y}, S U(2)_{L}$, and $S U(3)_{C}$, respectively.

Since the third generation Yukawa couplings are the dominant couplings in the superpotential (1), we shall retain only the elements $\left(h_{U}\right)_{33} \equiv h_{t},\left(h_{D}\right)_{33} \equiv h_{b},\left(h_{L}\right)_{33} \equiv h_{\tau}$ in each of the Yukawa couplings matrices $h_{U}, h_{D}, h_{L}$, setting all other elements equal to zero. Furthermore, since there are 36 independent $L$ violating trilinear couplings $\lambda_{a b c}$ and $\lambda_{a b c}^{\prime}$ in Eq. (2), and 9 independent $B$ violating couplings $\lambda_{a b c}^{\prime \prime}$ in the baryon number violating superpotential (3), we would have to consider 39 coupled nonlinear evolution equations for the $L$ violating case and 12 coupled nonlinear equations for the $B$ violating case, respectively. Thus, there is a clear need for a radical simplification of the evolution equations before we can study the RG evolution of the Yukawa couplings in the MSSM with $B$ and $L$ violation. Motivated by the generational hierarchy of the conventional Higgs couplings, we shall assume that an analogous hierarchy amongst the different generations of $B$ and $L$ violating couplings exists. Thus, we shall retain only the couplings $\lambda_{233}, \lambda_{333}^{\prime}, \lambda_{233}^{\prime \prime}$, and neglect the rest [9]. We note that $B$ and $L$ violating couplings to higher generations evolve more strongly because of larger Higgs couplings in their evolution equations, and hence could take larger values than the corresponding couplings to the lighter generations. We also note that the experimental upper limits are stronger for the $B$ and $L$ violating couplings with lower indices [11]. With these assumptions we can write the relevant renormalization group equations as [9]

$$
\begin{aligned}
16 & \pi^{2} \frac{d h_{t}^{2}}{d\left(-\ln \mu^{2}\right)} \\
& =h_{t}^{2}\left(\frac{16}{3} g_{3}^{2}+3 g_{2}^{2}+\frac{13}{15} g_{1}^{2}-6 h_{t}^{2}-h_{b}^{2}-\lambda_{333}^{\prime 2}-2 \lambda_{233}^{\prime \prime 2}\right),
\end{aligned}
$$

$$
\begin{aligned}
16 \pi^{2} & \frac{d h_{b}^{2}}{d\left(-\ln \mu^{2}\right)} \\
= & h_{b}^{2}\left(\frac{16}{3} g_{3}^{2}+3 g_{2}^{2}+\frac{7}{15} g_{1}^{2}-h_{t}^{2}-6 h_{b}^{2}-h_{\tau}^{2}\right. \\
& \left.-6 \lambda_{233}^{\prime 2}-2 \lambda_{233}^{\prime \prime 2}\right), \\
16 \pi^{2} & \frac{d h_{\tau}^{2}}{d\left(-\ln \mu^{2}\right)} \\
= & h_{\tau}^{2}\left(3 g_{2}^{2}+\frac{9}{5} g_{1}^{2}-3 h_{b}^{2}-4 h_{\tau}^{2}-4 \lambda_{233}^{2}-3 \lambda_{333}^{\prime 2}\right),
\end{aligned}
$$




$$
\begin{aligned}
16 \pi^{2} & \frac{d \lambda_{233}^{2}}{d\left(-\ln \mu^{2}\right)} \\
= & \lambda_{233}^{2}\left(3 g_{2}^{2}+\frac{9}{5} g_{1}^{2}-4 h_{\tau}^{2}-4 \lambda_{233}^{2}-3 \lambda_{233}^{\prime \prime 2}\right), \\
16 \pi^{2} \frac{d \lambda_{333}^{\prime 2}}{d\left(-\ln \mu^{2}\right)} & \lambda_{333}^{\prime 2}\left(\frac{16}{3} g_{3}^{2}+3 g_{2}^{2}+\frac{7}{15} g_{1}^{2}-h_{t}^{2}-6 h_{b}^{2}-h_{\tau}^{2}-\lambda_{233}^{2}\right. \\
& \left.-6 \lambda_{333}^{\prime 2}-2 \lambda_{333}^{\prime \prime 2}\right), \\
16 \pi^{2} & \frac{d \lambda_{233}^{\prime \prime 2}}{d\left(-\ln \mu^{2}\right)} \\
= & \lambda_{233}^{\prime \prime 2}\left(8 g_{3}^{2}+\frac{4}{5} g_{1}^{2}-2 h_{t}^{2}-2 h_{b}^{2}-2 \lambda_{333}^{\prime 2}-6 \lambda_{233}^{\prime \prime 2}\right),
\end{aligned}
$$

where $g_{1}, g_{2}, g_{3}$ are the gauge couplings of $U(1)_{Y}$ (in the GUT normalization), $S U(2)_{L}$ and $S U(3)_{C}$ gauge groups, respectively, and $\mu$ is the running mass scale. We note that within the context of grand unified theories, one is led to the situation where baryon and lepton number violating Yukawa couplings may be related at the GUT scale, and one may no longer be able to set one or the other arbitrarily to zero. We, therefore, include both, the baryon and the lepton number violating couplings, in our $R G$ equations. The evolution equations for the gauge couplings are not affected by the presence of $B$ and $L$ violating couplings at the one-loop level, and can be written, in the usual notation, as

$$
16 \pi^{2} \frac{d g_{i}^{2}}{d\left(-\ln \mu^{2}\right)}=-b_{i} g_{i}^{4}, \quad i=1,2,3
$$

with

$$
b_{i}=\left(\frac{33}{5}, 1,-3\right)
$$

The corresponding one-loop renormalization group equations for the gaugino masses $M_{i}, i=1,2,3$ can be written as

$$
16 \pi^{2} \frac{d M_{i}^{2}}{d\left(-\ln \mu^{2}\right)}=-2 b_{i} g_{i}^{2} M_{i}^{2} .
$$

Defining

$$
\begin{aligned}
& \widetilde{Y}_{i}=\frac{h_{i}^{2}}{16 \pi^{2}}, \quad i=t, b, \tau, \quad \tilde{Y}=\frac{\lambda_{233}^{2}}{16 \pi^{2}}, \\
& \tilde{Y}^{\prime}=\frac{\lambda_{333}^{\prime 2}}{16 \pi^{2}}, \quad \tilde{Y}^{\prime \prime}=\frac{\lambda_{233}^{\prime \prime 2}}{16 \pi^{2}},
\end{aligned}
$$

the solution of the RG equations (5)-(10) for the Yukawa and the $B$ and $L$ violating couplings can be written in a closed form [12]

$$
\widetilde{Y}_{k}(t)=\frac{\tilde{Y}_{k}(0) F_{k}(t)}{1+a_{k k} \tilde{Y}_{k}(0) \int_{0}^{t} F_{k}\left(t^{\prime}\right) d t^{\prime}}, \quad t=\ln \left(\frac{M_{X}^{2}}{\mu^{2}}\right)
$$

where $M_{X}$ is some large initial scale, and where $\widetilde{Y}_{k}$ stands for the functions $\widetilde{Y}_{i}(i=t, b, \tau), \widetilde{Y}, \widetilde{Y}^{\prime}$, and $\widetilde{Y}^{\prime \prime}$. Analogous notation holds for the functions $F_{k}$. The quantities $a_{k k}$ are the diagonal elements of the wave function anamolous dimension matrix, and are given by

$$
a_{k k}=\{6,6,4,4,6,6\},
$$

and the functions $F_{k}$ are given by the set of integral equations

$$
F_{t}(t)=\frac{E_{t}(t)}{\left(1+6 \widetilde{Y}_{b}(0) \int_{0}^{t} F_{b}\left(t^{\prime}\right) d t^{\prime}\right)^{1 / 6}\left(1+6 \widetilde{Y}^{\prime}(0) \int_{0}^{t} F^{\prime}\left(t^{\prime}\right) d t^{\prime}\right)^{1 / 6}\left(1+6 \widetilde{Y}^{\prime \prime}(0) \int_{0}^{t} F^{\prime \prime}\left(t^{\prime}\right) d t^{\prime}\right)^{1 / 3}},
$$

$$
F_{b}(t)=\frac{E_{b}(t)}{\left(1+6 \widetilde{Y}_{t}(0) \int_{0}^{t} F_{t}\left(t^{\prime}\right) d t^{\prime}\right)^{1 / 6}\left(1+4 \widetilde{Y}_{\tau}(0) \int_{0}^{t} F_{\tau}\left(t^{\prime}\right) d t^{\prime}\right)^{1 / 4}\left(1+6 \widetilde{Y}^{\prime}(0) \int_{0}^{t} F^{\prime}\left(t^{\prime}\right) d t^{\prime}\right)^{1 / 6}\left(1+6 \widetilde{Y}^{\prime \prime}(0) \int_{0}^{t} F^{\prime \prime}\left(t^{\prime}\right) d t^{\prime}\right)^{1 / 3}}
$$

$F_{\tau}(t)=\frac{E_{\tau(t)}}{\left(1+6 \widetilde{Y}_{b}(0) \int_{0}^{t} F_{b}\left(t^{\prime}\right) d t^{\prime}\right)^{1 / 2}\left(1+6 \widetilde{Y}(0) \int_{0}^{t} F\left(t^{\prime}\right) d t^{\prime}\right)\left(1+6 \widetilde{Y}^{\prime}(0) \int_{0}^{t} F^{\prime}\left(t^{\prime}\right) d t^{\prime}\right)^{1 / 2}}$,

$$
F(t)=\frac{E(t)}{\left(1+4 \widetilde{Y}_{\tau}(0) \int_{0}^{t} F_{\tau}\left(t^{\prime}\right) d t^{\prime}\right)\left(1+6 \tilde{Y}^{\prime \prime}(0) \int_{0}^{t} F^{\prime \prime}\left(t^{\prime}\right) d t^{\prime}\right)^{1 / 2}},
$$




$$
\begin{aligned}
F^{\prime}(t)= & \frac{E^{\prime}(t)}{\left(1+6 \widetilde{Y}_{t}(0) \int_{0}^{t} F_{t}\left(t^{\prime}\right) d t^{\prime} i\right)^{1 / 6}\left(1+6 \widetilde{Y}_{b}(0) \int_{0}^{t} F_{t}\left(t^{\prime}\right) d t^{\prime}\right)\left(1+4 \widetilde{Y}_{\tau}(0) \int_{0}^{t} F_{\tau}\left(t^{\prime}\right) d t^{\prime}\right)^{1 / 4}\left(1+4 \widetilde{Y}(0) \int_{0}^{t} F\left(t^{\prime}\right) d t^{\prime}\right)^{1 / 4}} \\
& \times \frac{1}{\left(1+6 \widetilde{Y}^{\prime \prime}(0) \int_{0}^{t} F^{\prime \prime}\left(t^{\prime}\right) d t^{\prime}\right)^{1 / 3}},
\end{aligned}
$$

$$
F^{\prime \prime}(t)=\frac{E^{\prime \prime}(t)}{\left(1+6 \widetilde{Y}_{t}(0) \int_{0}^{t} F_{t}\left(t^{\prime}\right) d t^{\prime}\right)^{1 / 3}\left(1+6 \widetilde{Y}_{b}(0) \int_{0}^{t} F_{t}\left(t^{\prime}\right) d t^{\prime}\right)^{1 / 3}\left(1+6 \widetilde{Y}^{\prime}(0) \int_{0}^{t} F^{\prime}\left(t^{\prime}\right) d t^{\prime}\right)^{1 / 3}},
$$

where the functions $E_{k}(t)\left[=E_{t}(t), E_{b}(t), E_{\tau}(t), E(t)\right.$, $E^{\prime}(t)$ and $\left.E^{\prime \prime}(t)\right]$ are given by

$$
E_{k}(t)=\prod_{i=1}^{3}\left(1+b_{i} \tilde{\alpha}_{i}(0) t\right)^{c_{k i} / b_{i}}
$$

with

$$
\begin{gathered}
\tilde{\alpha}_{i}(0)=\frac{g_{i}^{2}(0)}{16 \pi^{2}}, \quad i=1,2,3, \\
c_{t i}=\left(\frac{13}{15}, 3, \frac{16}{3}\right), \quad c_{b i}=\left(\frac{7}{15}, 3, \frac{16}{3}\right), \quad c_{\tau i}=\left(\frac{9}{5}, 3,0\right), \\
c_{\lambda_{233} i}=\left(\frac{9}{5}, 3,0\right), \quad c_{\lambda_{333}^{\prime} i}=\left(\frac{7}{15}, 3, \frac{16}{3}\right), \quad c_{\lambda_{233^{\prime \prime}} i}=\left(\frac{4}{5}, 0,8\right) .
\end{gathered}
$$

The solutions for the RG equations (11) for the gauge couplings and the gaugino masses (13) are well known and will not be repeated here. We note that Eq. (16) gives the exact solution for the Yukawa couplings, while $F_{k}$ 's in Eqs. (18)(23) should in principle be solved iteratively.

In order to study the infrared fixed points for the Yukawa couplings, it is convenient to redefine

$$
\begin{aligned}
& R_{t}=\frac{h_{t}^{2}}{g_{3}^{2}}, \quad R_{b}=\frac{h_{b}^{2}}{g_{3}^{2}}, \quad R_{\tau}=\frac{h_{\tau}^{2}}{g_{3}^{2}}, \quad R=\frac{\lambda_{233}^{2}}{g_{3}^{2}}, \\
& R^{\prime}=\frac{\lambda_{333}^{\prime 2}}{g_{3}^{2}}, \quad R^{\prime \prime}=\frac{\lambda_{233}^{\prime \prime 2}}{g_{3}^{2}},
\end{aligned}
$$

and retaining only the $S U(3)_{C}$ gauge coupling constant $g_{3}$, we can write the renormalization group equations (5)-(10) for the Yukawa couplings as

$3 g_{3}^{2} \frac{d R_{t}}{d g_{3}^{2}}=R_{t}\left[\frac{7}{3}-6 R_{t}-R_{b}-R^{\prime}-2 R^{\prime \prime}\right]$,

$$
\begin{aligned}
& 3 g_{3}^{2} \frac{d R_{b}}{d g_{3}^{2}}=R_{b}\left[\frac{7}{3}-R_{t}-6 R_{b}-R_{\tau}-6 R^{\prime}-2 R^{\prime \prime}\right], \\
& 3 g_{3}^{2} \frac{d R_{\tau}}{d g_{3}^{2}}=R_{\tau}\left[-3-3 R_{b}-4 R_{\tau}-4 R-3 R^{\prime}\right],
\end{aligned}
$$

$3 g_{3}^{2} \frac{d R}{d g_{3}^{2}}=R\left[-3-4 R_{\tau}-4 R-3 R^{\prime}\right]$

$3 g_{3}^{2} \frac{d R^{\prime}}{d g_{3}^{2}}=R^{\prime}\left[\frac{7}{3}-R_{t}-6 R_{b}-R_{\tau}-R-6 R^{\prime}-2 R^{\prime \prime}\right]$,

$3 g_{3}^{2} \frac{d R^{\prime \prime}}{d g_{3}^{2}}=R^{\prime \prime}\left[5-2 R_{t}-2 R_{b}-2 R^{\prime}-6 R^{\prime \prime}\right]$

There are no physically acceptable infrared fixed points for this set of RG equations with all the couplings attaining nontrivial values. The only nontrivial infrared fixed points are obtained by neglecting $R^{\prime}$. Then, the true infrared fixedpoints for these RG equations are [9]

$\left(R^{\prime *}, R_{b}^{*}, R_{t}^{*}\right)=\left(\frac{77}{102}, \frac{2}{17}, \frac{2}{17}\right), \quad R_{\tau}^{*}=R^{*}=0 \quad($ stable $)$,

$$
\begin{aligned}
& \left(R_{b}^{*}, R_{t}^{*}\right)=\left(\frac{1}{3}, \frac{1}{3}\right), \quad R_{\tau}^{*}=R^{*}=R^{\prime *}=0 \quad \text { (unstable) }, \\
& \left(R^{\prime *}, R_{t}^{*}\right)=\left(\frac{19}{24}, \frac{1}{8}\right), \quad R_{\tau}^{*}=R^{*}=R_{b}^{*}=0 \quad \text { (unstable), }
\end{aligned}
$$

$$
\left(R^{\prime \prime *}, R_{b}^{*}\right)=\left(\frac{19}{24}, \frac{1}{8}\right), \quad R_{\tau}^{*}=R^{*}=R_{t}^{*}=0 \quad \text { (unstable) }
$$

We note that the $\tau$ Yukawa coupling $R_{\tau}$ and the lepton number violating coupling $R$ approach trivial fixed point values 
since there is no contribution from the $S U(3)$ coupling $g_{3}$ in their renormalization group equations. Also, because of this reason the $B$ - and L-violating couplings do not approach simultaneous non-trivial fixed points. Furthermore, the infrared fixed point values of the top- and bottom-quark Yukawa couplings for the stable fixed point (35) are significantly different from the case when $B$ and $L$ is conserved [13]. Thus, the inclusion of $B$ and $L$ violation in MSSM has the effect of lowering the infrared fixed point values of the top- and bottom-quark Yukawa couplings. These are the important conclusions of our analysis. From Eq. (35) we note that the fixed point value for the top-quark Yukawa coupling translates into a top-quark (pole) mass of about $m_{t}$ $\simeq 70 \sin \beta \mathrm{GeV}$, which is incompatible with the measured value of [14] the top mass, $m_{t} \simeq 174 \mathrm{GeV}$, for any value of $\tan \beta$. Thus, the stable infrared fixed point (35) is not actually realized in nature.

The infrared fixed points (IRFP's) that we have discussed above are the true IRFP's of the renormalization equations for the Yukawa and baryon and lepton number violating cou- plings. However, these fixed points may not be reached in practice, the range between the large (GUT) scale and the weak scale being too small for the ratios to approach the fixed point values. In that case, the various Yukawa couplings may be determined by quasi-fixed point behavior [4], where the values of various couplings at the weak scale are independent of their values at the large scale, provided the Yukawa couplings at the large scale are large. More precisely, in the regime where the Yukawa couplings $\widetilde{Y}_{t}(0), \widetilde{Y}(0), \widetilde{Y}_{\tau}(0), \widetilde{Y}(0), \widetilde{Y}^{\prime}(0), \widetilde{Y}^{\prime \prime}(0) \rightarrow \infty$ with their ratios fixed, it is legitimate to drop 1 in the denominators of Eqs. (16) and (18)-(23) so that the exact solutions for the Yukawa couplings approach the infrared quasi-fixed-point (IRQFP) defined by

$$
\widetilde{Y}_{k}^{Q F P}(t)=\frac{F_{k}^{Q F P}(t)}{a_{k k} \int_{0}^{t} F_{k}^{Q F P}\left(t^{\prime}\right) d t^{\prime}},
$$

with

$$
\begin{aligned}
& F_{t}^{Q F P}(t)=\frac{E_{t}(t)}{\left(\int_{0}^{t} F_{b}^{Q F P}\left(t^{\prime}\right) d t^{\prime}\right)^{1 / 6}\left(\int_{0}^{t} F^{\prime Q F P}\left(t^{\prime}\right) d t^{\prime}\right)^{1 / 6}\left(\int_{0}^{t} F^{\prime \prime Q F P}\left(t^{\prime}\right) d t^{\prime}\right)^{1 / 3}}, \\
& F_{b}^{Q F P}(t)=\frac{E_{b}(t)}{\left(\int_{0}^{t} F_{t}^{Q F P}\left(t^{\prime}\right) d t^{\prime}\right)^{1 / 6}\left(\int_{0}^{t} F_{\tau}^{Q F P}\left(t^{\prime}\right) d t^{\prime}\right)^{1 / 4}\left(\int_{0}^{t} F^{\prime} Q F P\left(t^{\prime}\right) d t^{\prime}\right)^{1 / 6}\left(\int_{0}^{t} F^{\prime \prime Q F P}\left(t^{\prime}\right) d t^{\prime}\right)^{1 / 3}}, \\
& F_{\tau}^{Q F P}(t)=\frac{E_{\tau(t)}}{\left(\int_{0}^{t} F_{b} Q F P\left(t^{\prime}\right) d t^{\prime}\right)^{1 / 2}\left(\int_{0}^{t} F^{Q F P}\left(t^{\prime}\right) d t^{\prime}\right)\left(\int_{0}^{t} F^{\prime} Q F P\left(t^{\prime}\right) d t^{\prime}\right)^{1 / 2}}, \\
& F^{Q F P}(t)=\frac{E(t)}{\left(\int_{0}^{t} F_{\tau}^{Q F P}\left(t^{\prime}\right) d t^{\prime}\right)\left(\int_{0}^{t} F^{\prime \prime} Q F P\left(t^{\prime}\right) d t^{\prime}\right)^{1 / 2}} \\
& F^{\prime} Q F P(t)=\frac{E^{\prime}(t)}{\left(\int_{0}^{t} F_{t}^{Q F P}\left(t^{\prime}\right) d t^{\prime}\right)^{1 / 6}\left(\int_{0}^{t} F_{t}^{Q F P}\left(t^{\prime}\right) d t^{\prime}\right)\left(\int_{0}^{t} F_{\tau}^{Q F P}\left(t^{\prime}\right) d t^{\prime}\right)^{1 / 4}\left(\int_{0}^{t} F^{Q F P}\left(t^{\prime}\right) d t^{\prime}\right)^{1 / 4}\left(\int_{0}^{t} F^{\prime \prime Q F P}\left(t^{\prime}\right) d t^{\prime}\right)^{1 / 3}}, \\
& F^{\prime \prime Q F P}(t)=\frac{E^{\prime \prime}(t)}{\left(\int_{0}^{t} F_{t}\left(t^{\prime}\right) d t^{\prime}\right)^{1 / 3}\left(\int_{0}^{t} F_{t}\left(t^{\prime}\right) d t^{\prime}\right)^{1 / 3}\left(\int_{0}^{t} F^{\prime}\left(t^{\prime}\right) d t^{\prime}\right)^{1 / 3}} .
\end{aligned}
$$

We note that the result (39) follows immediately by dropping 1 from the denominator of Eq. (16), whereas the results (40)-(45) follow from the corresponding equations (18)(23) by using an iterative procedure $[12,15]$. We stress here that both the dependence on the initial conditions for each
Yukawa coupling as well as the dependence on the ratios of initial values of Yukawa couplings have completely dropped out of the runnings in Eqs. (39) and (40)-(45). In other words, the quasi-fixed points (39) are independent of whether the $B$ and $L$ violating couplings and the third gen- 
eration Yukawa couplings are unified or not. The fact that the ratios of the various Yukawa couplings do not enter Eqs. (39) -(45) implies that these results are valid for any $\tan \beta$ regime.

\section{B. Infrared fixed points for the trilinear soft supersymmetry breaking parameters}

We now consider the evolution equations for the soft supersymmetry breaking trilinear parameters in the potential (4). For these trilinear parameters we shall assume the same kind of generational hierarchy as was assumed for the corresponding Yukawa couplings. Thus, we shall consider only the highest generation trilinear couplings $\left(A_{U}\right)_{33} \equiv A_{t}$, $\left(A_{D}\right)_{33} \equiv A_{b}, \quad\left(A_{L}\right)_{33} \equiv A_{\tau}, \quad\left(A_{\lambda}\right)_{233} \equiv A_{\lambda}, \quad\left(A_{\lambda^{\prime}}\right)_{333} \equiv A_{\lambda^{\prime}}$, $\left(A_{\lambda^{\prime \prime}}\right)_{233} \equiv A_{\lambda^{\prime \prime}}$, setting all other elements equal to zero. As there is only one IRSFP (35) in the MSSM with $B$ and $L$ violation, we shall consider the IRFPs for the $A$ parameters corresponding to this case only, i.e. for $A_{t}, A_{b}, A_{\tau}, A_{\lambda}$ and $A_{\lambda^{\prime \prime}}$. Retaining only these parameters, and defining the ratios $\widetilde{A}_{i}=A_{i} / M_{3}\left(A_{i}=A_{t}, A_{b}, A_{\tau}, A_{\lambda}, A_{\lambda^{\prime \prime}}\right)$, we can write the relevant renormalization group equations [9] for $\widetilde{A}_{i}$ as [neglecting the $S U(2)_{L}$ and $U(1)_{Y}$ gauge couplings]

$$
\begin{aligned}
& 3 g_{3}^{2} \frac{d \widetilde{A}_{t}}{d g_{3}^{2}}=\left[\frac{16}{3}-\left(6 R_{t}+3\right) \widetilde{A}_{t}-R_{b} \widetilde{A}_{b}-2 R^{\prime \prime} \widetilde{A}_{\lambda^{\prime \prime}}\right] \\
& 3 g_{3}^{2} \frac{d \widetilde{A}_{b}}{d g_{3}^{2}}=\left[\frac{16}{3}-R_{t} \widetilde{A}_{t}-\left(6 R_{b}+3\right) \widetilde{A}_{b}-R_{\tau} \widetilde{A}_{\tau}-2 R^{\prime \prime} \widetilde{A}_{\lambda^{\prime \prime}}\right] \\
& 3 g_{3}^{2} \frac{d \widetilde{A}_{\tau}}{d g_{3}^{2}}=\left[-3 R_{b} \widetilde{A}_{b}-\left(4 R_{\tau}+\frac{R}{2}+3\right) \widetilde{A}_{\tau}-\frac{7}{2} R \widetilde{A}_{\lambda}\right] \\
& 3 g_{3}^{2} \frac{d \widetilde{A}_{\lambda}}{d g_{3}^{2}}=\left[-\frac{7}{2} R_{\tau} \widetilde{A}_{\tau}-\left(\frac{R_{\tau}}{2}+4 R+3\right) \widetilde{A}_{\lambda}\right]
\end{aligned}
$$

$$
3 g_{3}^{2} \frac{d \widetilde{A}_{\lambda^{\prime \prime}}}{d g_{3}^{2}}=\left[8-2 R_{t} \widetilde{A}_{t}-2 R_{b} \widetilde{A}_{b}-\left(6 R^{\prime \prime}+3\right) \widetilde{A}_{\lambda^{\prime \prime}}\right] .
$$

One can obtain the exact solutions for RG equations (46)(50) for the trilinear parameters $A_{i}$ analogous to the solutions (16) that we have obtained for the Yukawa couplings. The expressions for these solutions are lengthy, and will not be written down here. Instead we shall obtain approximate analytical solutions for these RG equations in Sec. III, and shall further study them numerically in Sec. IV. Nevertheless, we can obtain the true fixed points for the $\widetilde{A}_{i}$ parameters easily. These are

$$
\left(\widetilde{A}_{\lambda^{\prime \prime}}^{*}, \widetilde{A}_{b}^{*}, \widetilde{A}_{t}^{*}, \widetilde{A}_{\tau}^{*}\right)=\left(1,1,1,-\frac{2}{17}\right), \quad \widetilde{A}_{\lambda}^{*}=0 .
$$

It is straightforward to show that this is the only infrared stable fixed point for the $\widetilde{A}_{i}$ parameters. The stability of the fixed point (51) also follows from the general results connecting the stability of a set of $A$ parameters to the stability of the corresponding set of Yukawa couplings [16]. We further note that the fixed point values for $\widetilde{A}_{b}$ and $\widetilde{A}_{t}$ are the same as in MSSM with baryon number conservation [16]. However, the fixed point value for $\widetilde{A}_{\tau}$ is affected by the presence of the $B$-violating parameter $\widetilde{A}_{\lambda^{\prime \prime}}$.

\section{ANALYTICAL SOLUTIONS}

Having obtained the infrared fixed points of the renormalization group equations for the Yukawa couplings and the soft supersymmetry breaking trilinear couplings, it is important to determine the approach to these fixed points. The rate of approach to the fixed points can be determined by solving the RG equations in the neighborhood of the fixed points. Linearization of the RG equations (29)-(34) in the neighborhood of the stable infrared fixed point (35) leads to the following approximate analytical solution for the Yukawa and $B$ and $L$ violating couplings:

$$
\begin{aligned}
R_{t}= & \frac{2}{17}-0.002 R_{\tau 0}\left(\frac{g_{3}^{2}}{g_{30}^{2}}\right)^{-1}+\left[0.5\left(R_{t 0}-R_{b 0}\right)+0.02 R_{\tau 0}\right]\left(\frac{g_{3}^{2}}{g_{30}^{2}}\right)^{-1.18}+\left[0.48\left(R_{t 0}+R_{b 0}\right)-0.02 R_{\tau 0}-0.06 R_{0}^{\prime \prime}-0.07\right]\left(\frac{g_{3}^{2}}{g_{30}^{2}}\right)^{-0.2} \\
& +\left[0.02\left(R_{t 0}+R_{b 0}\right)+0.002 R_{\tau 0}+0.06 R_{0}^{\prime \prime}-0.05\right]\left(\frac{g_{3}^{2}}{g_{30}^{2}}\right)^{-1.68}, \\
R_{b}= & \frac{2}{17}+0.04 R_{\tau 0}\left(\frac{g_{3}^{2}}{g_{30}^{2}}\right)^{-1}-\left[0.5\left(R_{t 0}-R_{b 0}\right)+0.02 R_{\tau 0}\right]\left(\frac{g_{3}^{2}}{g_{30}^{2}}\right)^{-1.18}+\left[0.48\left(R_{t 0}+R_{b 0}\right)-0.02 R_{\tau 0}-0.06 R_{0}^{\prime \prime}-0.07\right]\left(\frac{g_{3}^{2}}{g_{30}^{2}}\right)^{-0.2} \\
& +\left[0.02\left(R_{t 0}+R_{b 0}\right)+0.002 R_{\tau 0}+0.06 R_{0}^{\prime \prime}-0.05\right]\left(\frac{g_{3}^{2}}{g_{30}^{2}}\right)^{-1.68},
\end{aligned}
$$




$$
\begin{aligned}
R_{\tau}= & R_{\tau 0}\left(\frac{g_{3}^{2}}{g_{30}^{2}}\right)^{-1}, \\
R= & R_{0}\left(\frac{g_{3}^{2}}{g_{30}^{2}}\right)^{-0.2}, \\
R^{\prime \prime}= & \frac{77}{102}-0.005 R_{\tau 0}\left(\frac{g_{3}^{2}}{g_{30}^{2}}\right)^{-1}+\left[-0.37\left(R_{t 0}+R_{b 0}\right)+0.02 R_{\tau 0}+0.05 R_{0}^{\prime \prime}+0.05\right]\left(\frac{g_{3}^{2}}{g_{30}^{2}}\right)^{-0.2}+\left[0.37\left(R_{t 0}+R_{b 0}\right)+0.03 R_{\tau 0}\right. \\
& \left.+0.95 R_{0}^{\prime \prime}-0.8\right]\left(\frac{g_{3}^{2}}{g_{30}^{2}}\right)^{-1.68},
\end{aligned}
$$

where $R_{t 0}, R_{b 0}, R_{\tau 0}, R_{0}, R_{0}^{\prime \prime}$ are the corresponding initial values at the large (UV) scale. These equations determine, in the leading order, how fast the infrared fixed point (35) is approached. The measure of infrared attraction is the size of the negative power in $\left(g_{3}^{2} / g_{30}^{2}\right)$ in various terms in the expression for $R_{i}$.

Similarly, linearization of the RG equations (46)-(50) for the trilinear couplings about the stable infrared fixed point (51) leads to the following approximate solution for these couplings:

$$
\begin{aligned}
\widetilde{A}_{t}= & 1+0.5\left(\widetilde{A}_{t 0}-\widetilde{A}_{b 0}\right)\left(\frac{g_{3}^{2}}{g_{30}^{2}}\right)^{-1.2}+0.4\left(\widetilde{A}_{\lambda^{\prime \prime} 0}-1\right)\left(\frac{g_{3}^{2}}{g_{30}^{2}}\right)^{-2.57}-0.5\left(1.2-\left(\widetilde{A}_{t 0}+\widetilde{A}_{b 0}\right)+0.8 \widetilde{A}_{\lambda^{\prime \prime}}\right)\left(\frac{g_{3}^{2}}{g_{30}^{2}}\right)^{-1.21}, \\
\widetilde{A}_{b}= & 1-0.5\left(\widetilde{A}_{t 0}-\widetilde{A}_{b 0}\right)\left(\frac{g_{3}^{2}}{g_{30}^{2}}\right)^{-1.2}+0.4\left(\widetilde{A}_{\lambda^{\prime \prime} 0}-1\right)\left(\frac{g_{3}^{2}}{g_{30}^{2}}\right)^{-2.57}-0.5\left(1.2-\left(\widetilde{A}_{t 0}+\widetilde{A}_{b 0}\right)+0.8 \widetilde{A}_{\lambda^{\prime \prime} 0}\right)\left(\frac{g_{3}^{2}}{g_{30}^{2}}\right)^{-1.21}, \\
\widetilde{A}_{\tau}= & -\frac{2}{17}-0.3\left(\widetilde{A}_{t 0}-\widetilde{A}_{b 0}\right)\left(\frac{g_{3}^{2}}{g_{30}^{2}}\right)^{-1.2}+0.03\left(\widetilde{A}_{\lambda^{\prime \prime} 0}-1\right)\left(\frac{g_{3}^{2}}{g_{30}^{2}}\right)^{-2.57}+\left(0.47+0.03 \widetilde{A}_{t 0}-0.57 \widetilde{A}_{b 0}+\widetilde{A}_{\tau 0}+0.19 \widetilde{A}_{\lambda^{\prime \prime} 0}\right) \\
& \times\left(\frac{g_{3}^{2}}{g_{30}^{2}}\right)^{-1}-0.27\left(1.2-\left(\widetilde{A}_{t 0}+\widetilde{A}_{b 0}\right)+0.8 \widetilde{A}_{\lambda^{\prime \prime} 0}\right)\left(\frac{g_{3}^{2}}{g_{30}^{2}}\right)^{-1.21}, \\
\widetilde{A}_{\lambda}= & \widetilde{A}_{\lambda 0}\left(\frac{g_{3}^{2}}{g_{30}^{2}}\right)^{-1}, \\
\widetilde{A}_{\lambda^{\prime \prime}}= & 1+\left(\widetilde{A}_{\lambda^{\prime \prime} 0}-1\right)\left(\frac{g_{3}^{2}}{g_{30}^{2}}\right)^{-2.57}+0.06\left(1.2-\left(\widetilde{A}_{t 0}+\widetilde{A}_{b 0}\right)+0.8 \widetilde{A}_{\lambda^{\prime \prime} 0}\right)\left(\frac{g_{3}^{2}}{g_{30}^{2}}\right)^{-1.21},
\end{aligned}
$$

where $\widetilde{A}_{t 0}, \widetilde{A}_{b 0}, \widetilde{A}_{\tau 0}, \widetilde{A}_{\lambda 0}, \widetilde{A}_{\lambda^{\prime \prime} 0}$ are the initial values of the $A$ parameters at the UV scale.

\section{NUMERICAL RESULTS}

Having obtained analytically the true fixed points as well as the quasi-fixed points for the various couplings, as well as the approximate solutions to the corresponding RG equations, it is instructive to carry out a numerical study of these equations in order to illustrate the discussion of the previous sections.

Since the RG equations are coupled differential equations with several fixed points, this implies that the system of equations (29)-(34) has an infrared fixed surface, the $R_{t}$ $-R_{b}-R^{\prime \prime}$ surface, $R_{\tau}=R=0$. In principle, a three dimensional plot for the evolutions would reveal the infra-red fixed surfaces, with the sole infrared stable fixed point being approached by the flow from different directions. However, such a demonstration is rather cumbersome. We shall instead concentrate on the two dimensional projections of this three dimensional surface to illustrate the approach to the fixed point.

For the purposes of our numerical analysis, we set the unification scale $M_{X} \simeq 10^{16} \mathrm{GeV}$, the unified gauge coupling $\alpha_{G}\left(M_{X}\right)=1 / 24.5$ and an effective supersymmetry scale of 
$M_{S}=1 \mathrm{TeV}$. We shall use the one-loop RG equations in the numerical analysis, since the difference between the oneloop and two-loop results for the infrared fixed points [17] is less than $10 \%$. Reinserting the electroweak gauge couplings $g_{1}, g_{2}$ in the RG equations (29)-(34), the fixed points are no longer found to be exact. In order to determine the approximate positions of the fixed points, we shall maintain running $g_{1}(\mu), g_{2}(\mu)$ and follow the prescription that the approximate infrared fixed point in the $R_{t}-R_{b}-R^{\prime \prime}$ space is determined as the unique point in the evolution path from $\mu$ $=M_{X}$ to $\mu=M_{S}$ which has the same value at $\mu=M_{X}$ and at $\mu=M_{S}$. The results depend somewhat on the evolution path, and in particular on the scale $M_{X}$, the deviations from the values in Eq. (35) being larger, the larger the scale $M_{X}$. We note that the idea of grand unification is not important for our results. The infrared fixed point and the infrared fixed surface are the properties of minimal supersymmetric standard model with baryon and lepton number violation. Note that it is only in that they are not exact but approximate do they depend on $M_{X}$.

Our main objective here is to study the renormalization group flow in the coupling ratios $R_{t}, R_{b}, R^{\prime \prime}$ from $M_{X}$ to $M_{S}$, to display the fixed surfaces (projections of three dimensional surface onto two dimensions), and to locate the infrared fixed point. We start with $h_{t}$ and $h_{b}$ each taking the values $(9,1,0.5)$ to ensure a large range in the variation of these parameters and $\lambda_{233}^{\prime \prime}$ fixed at a reasonably large value of 1.1 for the computations that we perform. We evolve the Yukawa couplings down to the IR scale using the one-loop $\mathrm{RG}$ equations and evaluate the coupling ratios $R_{t}, R_{b}, R^{\prime \prime}$. The values of the couplings so obtained at the IR scale, viz., $M_{S}$, are then used as the input at the UV scale, $M_{X}$, and evolved down to the IR scale and then iterated again. This procedure is repeated until all the final points meet in the infrared fixed point. The results of these iterations are then projected onto the $R_{t}-R_{b}, R_{t}-R^{\prime \prime}$ and $R_{b}-R^{\prime \prime}$ planes, respectively. The results so obtained are presented in Figs. 1(a), 1(b) and 1(c), respectively. It may be observed that the approach to the fixed point of the ratio $R^{\prime \prime}$ is rather rapid since at the outset this quantity was chosen close to it. The approach of the ratios $R_{t}$ and $R_{b}$ is significantly more dramatic since they are required to approach the fixed point from rather disparate values. This clearly illustrates the strong attraction to the sole IRSFP.

The end result of the series of iterations that we have performed lead to the following infrared fixed point values for the coupling ratios:

$$
\begin{gathered}
R^{\prime Q F P} \simeq 0.65, \\
R_{b}^{Q F P} \simeq 0.59, \\
R_{t}^{Q F P} \simeq 0.59 .
\end{gathered}
$$

This infrared fixed point and the fixed surfaces are as expected from the preceeding analytical considerations with, however, shifted position of the fixed point due to the effect of including the electroweak gauge couplings in our numerical iterations. It is, in fact, the quasi fixed point for the re-
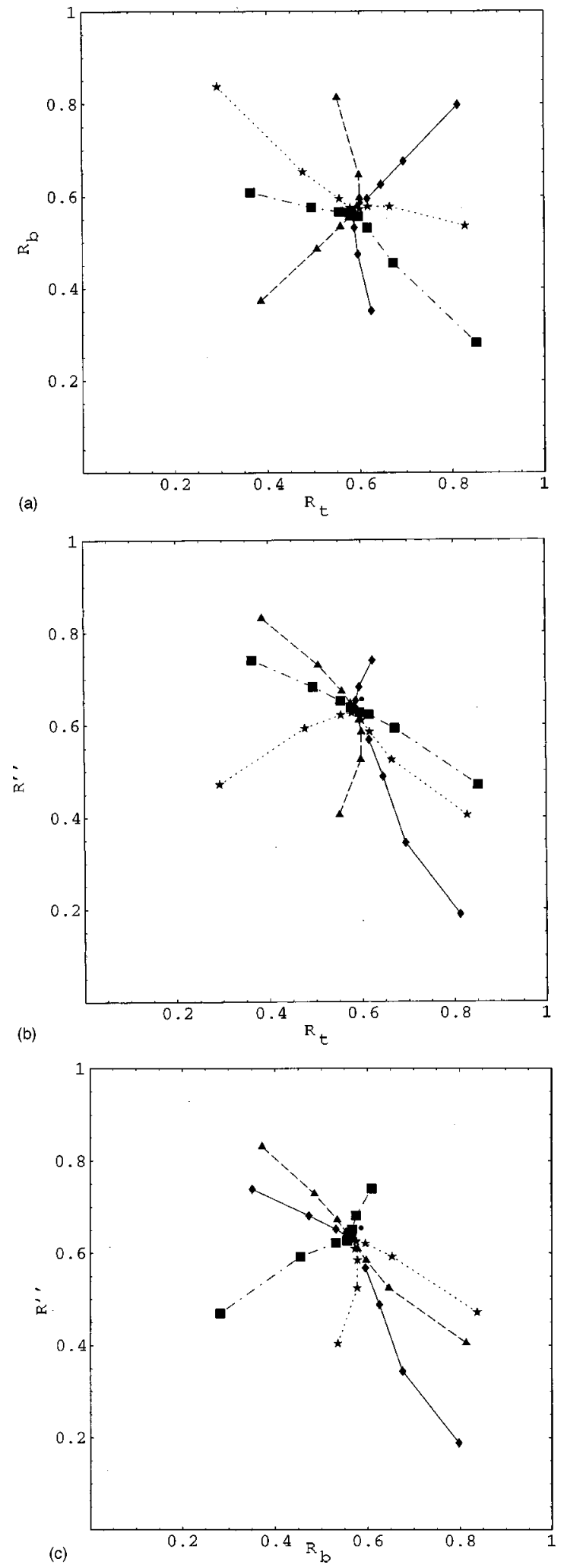

FIG. 1. (a) The values of the ratios $R_{b}$ and $R_{t}$ at the end of first four iterations for the inputs $h_{t}=9,1,0.5, h_{b}=9,1,0.5$, and $\lambda_{233}^{\prime \prime}$ $=1.1$. (b) The values of the ratios $R^{\prime \prime}$ and $R_{t}$ at the end of first four iterations for the inputs $h_{t}=9,1,0.5, h_{b}=9,1,0.5$, and $\lambda_{233}^{\prime \prime}=1.1$. (c) The values of the ratios $R^{\prime \prime}$ and $R_{b}$ at the end of first four iterations for the inputs $h_{t}=9,1,0.5, h_{b}=9,1,0.5$, and $\lambda_{233}^{\prime \prime}=1.1$. 
spective coupling ratios. The fixed point (62) implies the approximate top-bottom Yukawa unification at all scales $\mu$.

The fixed point values in Eq. (62) translate into the fixed point values for the top-, bottom-quark Yukawa couplings, and the baryon number violating coupling:

$$
\begin{gathered}
\lambda_{233}^{\prime Q F P} \simeq 0.90, \\
h_{b}^{Q F P} \simeq 0.86, \\
h_{t}^{Q F P} \simeq 0.86 .
\end{gathered}
$$

These quasi-fixed-point values for the Yukawa couplings are not significantly different from those obtained in a situation when the $\tau$ Yukawa coupling was ignored, and when the baryon and lepton number violating couplings were considered separately in the fixed point analysis [7]. Since the quasi-fixed points are reached for large initial values of the couplings at the GUT scale, these reflect on the assumption of perturbative unitarity, or the absence of Landau poles, of the corresponding couplings. The quasi-fixed points (63), therefore, provide an upper bound on the relevant Yukawa and the baryon and lepton number violating couplings. From our analysis we, thus, conclude that $\lambda_{233}^{\prime \prime} \lesssim 1$ in a process independent manner.

We have carried an analogous numerical study for the soft supersymmetry breaking trilinear couplings. Here the choice of inputs is larger and we economize on the possibilities, however, ensuring that the main focus of the analysis is not lost. In Figs. 2(a), 2(b) and 2(c) we illustrate the infrared attraction for the trilinear couplings with a choice of Yukawa and the baryon number violating couplings $h_{b}=\lambda_{233}^{\prime \prime}=1.1$ and $h_{t}=1.65$ (in order to break the near isospin invariance of the top- and bottom-quark couplings), and universal boundary conditions for the trilinear couplings $\pm(3,2,1,0.5) M_{1 / 2}$, 0 , with $M_{1 / 2}$ the universal gaugino mass, for the eight different computations that we perform. The results for the Yukawa couplings and the trilinear couplings at the infrared scale are then fed back to the RG equations at the UV scale and then evolved again. We exhibit the resulting attraction in terms of the projections onto the $\widetilde{A}_{t}-\widetilde{A}_{b}, \widetilde{A}_{t}-\widetilde{A}_{\lambda^{\prime \prime}}$ and $\widetilde{A}_{b}$ $-\widetilde{A}_{\lambda^{\prime \prime}}$ planes in Fig. 2. The dramatic focusing property of the renormalization group equations for the $A$ parameters is clearly observed in these results. The results of this iteration process results in the following predictions for the quasifixed points for the $A$ parameters:

$$
\begin{aligned}
& A_{\lambda^{\prime \prime}}^{Q F P} \simeq 0.95 m_{\tilde{g}}, \\
& A_{t}^{Q F P} \simeq 0.66 m_{\tilde{g}}, \\
& A_{b}^{Q F P} \simeq 0.67 m_{\tilde{g}},
\end{aligned}
$$

where $m_{\tilde{g}}$ is the gluino mass $\left(=M_{3}\right)$ at the weak scale. These quasi-fixed point values for the $A$ parameters must be compared with the true fixed point values (51). We note that the quasi-fixed-point values (64) provide a lower bound on the corresponding $A$ parameters, whereas the true fixed-point values (51) represent an upper bound on these parameters.
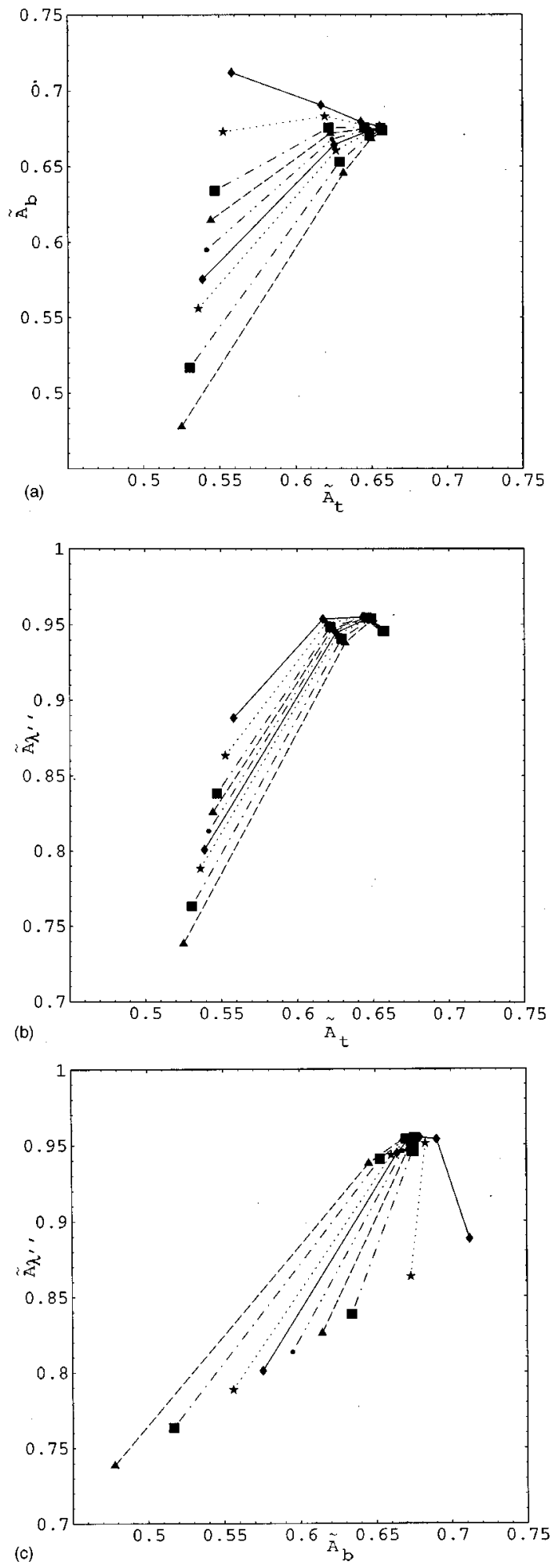

FIG. 2. (a) The values of the ratios $\widetilde{A}_{b}$ and $\widetilde{A}_{t}$ at the end of first four iterations for the inputs $h_{b}=\lambda_{233}^{\prime \prime}=1.1$ and $h_{t}=1.65$, and universal trilinear couplings $A= \pm(3,2,1,0.5) M_{1 / 2}, 0$. (b) The values of the ratios $\widetilde{A}_{\lambda^{\prime \prime}}$ and $\widetilde{A}_{t}$ at the end of first four iterations for the inputs $h_{b}=\lambda_{233}^{\prime \prime}=1.1$ and $h_{t}=1.65$, and universal trilinear couplings $A= \pm(3,2,1,0.5) M_{1 / 2}, 0$. (c) The values of the ratios $\widetilde{A}_{\lambda^{\prime \prime}}$ and $\widetilde{A}_{b}$ at the end of first four iterations for the inputs $h_{b}=\lambda_{233}^{\prime \prime}$ $=1.1$ and $h_{t}=1.65$, and universal trilinear couplings $A=$ $\pm(3,2,1,0.5) M_{1 / 2}, 0$. 
From this analysis we are able to constrain these $A$ parameters in the following model independent manner:

$$
\begin{aligned}
& \frac{A_{\lambda^{\prime \prime}}}{m_{g}} \simeq 1, \\
& 0.66 \lesssim \frac{A_{t}}{m_{\tilde{g}}} \lesssim 1, \\
& 0.67 \lesssim \frac{A_{b}}{m_{g}} \lesssim 1 .
\end{aligned}
$$

\section{SUMMARY AND CONCLUSIONS}

We have carried out a detailed study of the infrared fixed point structure of the minimal supersymmetric standard model with the third generation Yukawa couplings and with highest generation baryon and lepton number violation. We have obtained the infrared fixed points for such a model, and shown that there is no physically acceptable infrared fixed point with both baryon and lepton number violating couplings approaching a nontrivial fixed point. The simultaneous nontrivial fixed point for the top- and bottom-quark Yukawa couplings, and the $B$-violating coupling $\lambda_{233}^{\prime \prime}$ is the only true fixed point that is stable in the infrared region. We have obtained approximate analytical solutions to the RG equations near the stable fixed point, which illustrate the approach to the fixed point. We have also derived the exact solutions of the RG equations of such a model in a closed form, from which we have obtained infrared quasi-fixed point solutions for the various couplings. The quasi-fixedpoints are realized at the weak scale when the initial couplings at the GUT scale are large. These fixed points, thus, reflect on the assumption of the perturbative unitarity of the corresponding couplings. We have carried out the corresponding $\mathrm{RG}$ analysis of the trilinear soft supersymmetry breaking couplings, and obtained the infrared stable fixed point for these couplings, and the approach to the fixed point analytically.

Since the true fixed points may not be reached at the electroweak scale, we have also studied the numerical solutions of the RG equations, and obtained the infrared fixed surfaces, and demonstrated the convergence of the RG flow towards the fixed point. From this analysis we have obtained a process independent upper bound on the highest generation baryon number violating coupling, $\lambda_{233}^{\prime \prime} \lesssim 1$.

Our study of the RG flow of the Yukawa couplings has been complemented by the corresponding numerical study of the RG equations for the soft supersymmetry breaking trilinear couplings, and the demonstration of the rapid convergence towards the fixed point for these couplings. From this analysis, we have constrained the $A$ parameters to be $A_{\lambda^{\prime \prime}} / m_{\tilde{g}} \simeq 1,0.66 \lesssim A_{t} / m_{\tilde{g}} \lesssim 1,0.67 \lesssim A_{b} / m_{\tilde{g}} \lesssim 1$. We emphasize that our results are independent of whether or not there is a unification of the couplings at some large scale or not. The infrared fixed points and the infrared fixed surfaces are the property of the MSSM with baryon and lepton number violation. Since they are approximate, only their actual value depends on the large scale.

\section{ACKNOWLEDGMENTS}

We thank the organizers of the WHEPP6, the Sixth Workshop on High Energy Particle Physics, held in Chennai, India for their hospitality during which this work was started. B.A. thanks the Institut für Theoretische Physik, Universität Bern, Switzerland for its hospitality when part of this work was done. The work of P.N.P. is supported by the University Grants Commission Research Grant No. F.30-63/98 (SA III). He would like to thank the Inter-University Center for Astronomy and Astrophysics, Pune, India for its hospitality while part of this work was done.
[1] B. Schrempp and M. Wimmer, Prog. Part. Nucl. Phys. 37, 1 (1996).

[2] B. Pendleton and G. G. Ross, Phys. Lett. 98B, 291 (1981).

[3] M. Lanzagorta and G. G. Ross, Phys. Lett. B 349, 319 (1995).

[4] C. T. Hill, Phys. Rev. D 24, 691 (1981).

[5] S. Weinberg, Phys. Rev. D 26, 287 (1982); N. Sakai and T. Yanagida, Nucl. Phys. B197, 533 (1982).

[6] G. R. Farrar and P. Fayet, Phys. Lett. 76B, 575 (1978).

[7] V. Barger, M. S. Berger, R. J. Phillips, and T. Wohrmann, Phys. Rev. D 53, 6407 (1996); see also B. Brahmachari and P. Roy, ibid. 50, 39 (1994).

[8] B. Ananthanarayan and P. N. Pandita, Phys. Lett. B 454, 84 (1999).
[9] B. Ananthanarayan and P. N. Pandita, Phys. Rev. D 62, 036009 (2000), and references therein.

[10] B. C. Allanach, A. Dedes, and H. K. Dreiner, Phys. Rev. D 60, 056002 (1999).

[11] R. Barbier et al., hep-ph/9810232.

[12] G. Auberson and G. Moultaka, Eur. Phys. J. C 12, 331 (2000).

[13] B. Schrempp, Phys. Lett. B 344, 193 (1995).

[14] Particle Data Group, D. E. Groom et al., Eur. Phys. J. C 15, 1 (2000).

[15] D. Kazakov and G. Moultaka, Nucl. Phys. B577, 121 (2000).

[16] I. Jack and D. R. T. Jones, Phys. Lett. B 443, 177 (1998).

[17] S. Codoban and D. I. Kazakov, Eur. Phys. J. C 13, 671 (2000). 